\title{
Quantized acoustoelectric current in the presence of large tunneling counterflow
}

\author{
Gloos, K.; Utko, P.; Hansen, Jørn Bindslev; Lindelof, Poul Erik
}

Published in:

Physical Review B Condensed Matter

Link to article, DOI:

10.1103/PhysRevB.70.235345

Publication date:

2004

Document Version

Publisher's PDF, also known as Version of record

Link back to DTU Orbit

Citation (APA):

Gloos, K., Utko, P., Hansen, J. B., \& Lindelof, P. E. (2004). Quantized acoustoelectric current in the presence of large tunneling counterflow. Physical Review B Condensed Matter, 70(23), 235345.

https://doi.org/10.1103/PhysRevB.70.235345

\section{General rights}

Copyright and moral rights for the publications made accessible in the public portal are retained by the authors and/or other copyright owners and it is a condition of accessing publications that users recognise and abide by the legal requirements associated with these rights.

- Users may download and print one copy of any publication from the public portal for the purpose of private study or research.

- You may not further distribute the material or use it for any profit-making activity or commercial gain

- You may freely distribute the URL identifying the publication in the public portal 


\title{
Quantized acoustoelectric current in the presence of large tunneling counterflow
}

\author{
K. Gloos, ${ }^{1}$ P. Utko, ${ }^{1}$ J. Bindslev Hansen, ${ }^{2}$ and P. E. Lindelof ${ }^{1}$ \\ ${ }^{1}$ Nano-Science Center, Niels Bohr Institute fAFG, University of Copenhagen, Universitetsparken 5, DK-2100 Copenhagen, Denmark \\ ${ }^{2}$ Department of Physics, Technical University of Denmark, DK-2800 Lyngby, Denmark \\ (Received 26 April 2004; revised manuscript received 19 July 2004; published 30 December 2004)
}

\begin{abstract}
A surface acoustic wave drives an electrical current through a short quantum wire. A second tunneling current is injected by biasing one side of the quantum wire. These two contributions to the total current, which flow in opposite directions, are controlled almost independently by the gate and the bias voltage, respectively. We have observed the quantization of the acoustoelectric current at up to ten times larger counterflowing tunneling currents. At large tunneling currents the acoustoelectric current can be strongly suppressed. However, this does not seem to be due to an electrostatic interaction between the two currents, but is probably caused by the complex potential landscape in the narrow channel of the quantum wire.
\end{abstract}

DOI: $10.1103 /$ PhysRevB.70.235345

PACS number(s): 73.63.Nm, 72.50.+b, 73.40.Gk, 06.20.Jr

\section{INTRODUCTION}

Dual transport of charge is well known in solid-state physics. ${ }^{1}$ The prime example is the two-band transport due to the existence of electron and hole Fermi surfaces in metals, semimetals, and semiconductors such as in bismuth. ${ }^{2}$ Other dual effects occur when carriers of the same type are transported by two different mechanisms. In the so-called ratchet effects, rectification of an applied voltage may change sign as the temperature is decreased because diffussive processes dominating at high temperatures are replaced by tunneling transport at low temperatures. ${ }^{3}$

Two electrical currents flowing near each other interact via Coulomb repulsion or by phonon transfer. This interaction is the stronger the shorter the distance between them is. An example of this would be the studies on frictional drag between two closely spaced two-dimensional electron gases (2DEG) in a magnetic field. ${ }^{4,5}$ Another example would be two closely spaced quantum wires. For such systems minimum distances down to several $1 \mathrm{~nm}$ have been achieved, for example, by the cleaved-edge overgrowth technique. ${ }^{6,7}$ In these cases, the large tunneling probability allows one to study the collective excitations of the electron population in the wires. ${ }^{7}$ The spatial separation ensures that the currents in these systems can be separated. In the ultimate limit this separation tends to zero when the two wires occupy the same spatial region. However, distinguishing the two different currents would then be almost impossible.

We have found a way to simultaneously generate, control, and observe two different electrical currents flowing through a short quantum wire, the channel of a quantum-point contact (QPC). One current is dragged by a dynamic surface acoustic wave (SAW) and the other one by a static potential difference across the QPC.

The QPC itself is defined in the 2DEG of a GaAs/AlGaAs heterostructure and kept in the closed regime below conductance pinch-off. Due to an applied bias voltage, hot electrons can tunnel from one side of the QPC to the other. A second current is driven by a SAW from the opposite side of the constriction up the potential hill of the QPC. The absolute value of both currents can be adjusted by the gate as well as the bias voltage, however, in a different manner. In particular, when the SAW is incident on the positively biased side of the QPC, the acoustoelectric current depends more sensitively on the bias voltage while the tunneling current is more sensitive to the gate voltage.

The tunneling current $I_{T}$ varies exponentially with bias and gate voltage. SAWs, on the other hand, transfer single (or few) electrons per SAW cycle across the potential hill of the QPC. ${ }^{8-11}$ This situation can be described by "moving quantum dots" traveling with sound velocity $v_{S A W} \approx 3 \mathrm{~km} / \mathrm{s}$ (Ref. 8). Coulomb repulsion between the electrons in such a dot $^{12}$ determines its occupancy as function of gate and bias voltage, SAW amplitude, and frequency. Thus the acoustoelectric (AE) current changes stepwise by developing plateaus at $I_{A E}=n e f$. Here $e$ is the electron charge; $f$, the SAW frequency; and $n$, an integer. Theoretical models attribute deviations from perfectly flat $I_{A E}$ plateaus to electron tunneling and thermal activation either out of ${ }^{12-14}$ or into the moving quantum dots. ${ }^{15}$ Since the AE current and the biasinduced tunneling current depend, in distinctively different ways, on bias and gate voltage, they can be separated at least over a certain range of parameters.

The outline of this paper is as follows: Sec. II describes the layout and fabrication of the three investigated samples. Section III shows their behavior in the presence of counterflowing acoustoelectric and tunneling currents. Section IV focuses on the QPC potential barrier as function of bias and gate voltage below pinch-off for conduction. A discussion follows on how to separate the two components of the total current as well as their possible interaction with each other. Section V summarizes the results.

\section{EXPERIMENTAL DETAILS}

Our samples were fabricated on a GaAs/AlGaAs heterostructure. ${ }^{16}$ Its 2DEG had a mobility of $105 \mathrm{~m}^{2} / \mathrm{V} \mathrm{s}$ and a carrier density of $2.8 \times 10^{15} \mathrm{~m}^{-2}$, measured in the dark at $10 \mathrm{~K}$. This corresponds to a Fermi energy of $\epsilon_{F}$ $\approx 10 \mathrm{meV}$ and a long electron mean-free path of $l \approx 9 \mu \mathrm{m}$.

The QPC was patterned by electron-beam lithography. Two semicircular shallow-etched trenches formed a smooth constriction between the two electron reservoirs, whereas the 

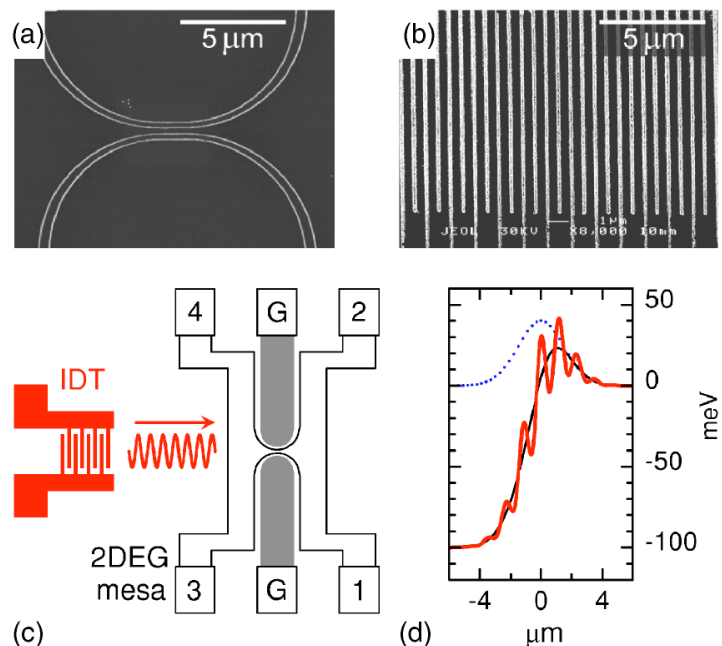

FIG. 1. (Color online) (a) Scanning-electron micrograph of the shallow etched constriction of sample 2B. (b) Section of the interdigital transducer (IDT). (c) Schematic sample layout: 2DEG mesa with the QPC, four Ohmic contacts (1-4) to the 2DEG, two side gates $(\mathrm{G})$, and the active IDT. In the experiments, the SAW is incident from the left, contact 3 is biased, and the current detected at contact 1, defining the virtual ground. (d) Schematic potential landscape through the center of the positively biased QPC along the propagation direction of the superposed SAW (thick solid line). The electrical component of the SAW is reduced outside the QPC due to screening by the 2DEG. Also shown is the potential of the unbiased constriction (dotted line) and of the positively biased QPC (thin solid line), both without SAW. In all cases, the right-hand side of the QPC is kept at virtual ground.

large areas of the 2DEG on both sides of the channel served as side gates [Fig. 1(a)]. The $200 \mathrm{~nm}$ wide and $40 \mathrm{~nm}$ deep trenches had curvature radii as indicated in Table I. Two of the samples had an additional $1 \mu \mathrm{m}$ long straight section in the center of the QPC. The geometrical width of the channel formed by the two trenches was about $200 \mathrm{~nm}$.

Two aluminum interdigital transducers (IDTs), each with 80 pairs of fingers [Fig. 1(b)], were deposited on both sides of a 2DEG mesa. Their fundamental acoustic wavelength $\lambda$ $=1.15 \mu \mathrm{m}$ corresponded to a center frequency of $f$ $=2.46 \mathrm{GHz}$. The distance between either of the two IDTs and the QPC was about $1.3 \mathrm{~mm}$. Only one of the IDTs was used

TABLE I. Basic QPC properties of the three investigated samples cut from wafer HCO103-92-30122. Each QPC was defined by shallow-etched trenches with the indicated curvature radius. Samples $2 \mathrm{~B}$ and $2 \mathrm{C}$ had an additional $1 \mu \mathrm{m}$ long straight segment in their center. The parameters $\alpha$ and $\beta$ describe how the barrier height changes with bias and gate voltage, respectively, as discussed in the text.

\begin{tabular}{ccccc}
\hline \hline Sample & $\begin{array}{c}\text { Radius } \\
(\mu \mathrm{m})\end{array}$ & $\begin{array}{c}\text { Segment } \\
(\mu \mathrm{m})\end{array}$ & $\alpha$ & $\beta$ \\
\hline 2B & 5.0 & 1.0 & 0.045 & 0.92 \\
$2 \mathrm{C}$ & 7.5 & 1.0 & 0.194 & 0.50 \\
$2 \mathrm{E}$ & 10.0 & 0.0 & 0.054 & 0.81 \\
\hline \hline
\end{tabular}

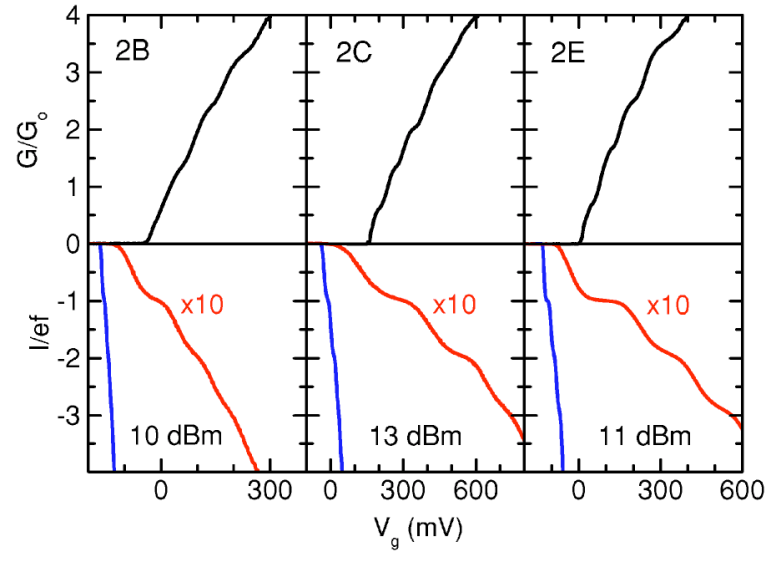

FIG. 2. (Color online) Typical characteristics of the three devices with respect to the gate voltage $V_{g}$ at $T=1.8 \mathrm{~K}$. The upper traces (SAW off) show the conductance $G$ normalized to the quantum conductance $G_{0}=2 e^{2} / h$. The data are corrected for a series resistance of $0.6 \mathrm{k} \Omega$. Lower traces (SAW on) are the AE current $I$ in units of $e f$. Also shown are the same current traces blown up along the $V_{g}$ axis by a factor of 10 to visualize the first few quantized current steps. The $\mathrm{rf}$ generator was set at the indicated power and frequencies of 2472.085 (2B), 2464.780 (2C), and $2466.565 \mathrm{MHz}$ (2E).

to launch the SAW, as indicated schematically in Fig. 1(c).

All measurements were carried out in vacuum at $1.8 \mathrm{~K}$ base temperature of the refrigerator. However, the rf power for generating the SAW raised the temperature of the $2 \mathrm{DEG}$ to about $5 \mathrm{~K}$. In addition to the total current $I$, we also recorded its derivatives with respect to the gate voltage $d I / d V_{g}$ (transconductance) and to the source-drain bias $d I / d V_{s d}$ (conductance). The excitation amplitude was $d V_{g}=0.5 \mathrm{mV}$ at $17 \mathrm{~Hz}$ and $d V_{s d}=0.5 \mathrm{mV}$ at $117 \mathrm{~Hz}$. Smaller excitation amplitudes of $0.2 \mathrm{mV}$ yielded the same results. Leakage to the gates was always negligible $\left(I_{l e a k} \ll 0.1 \mathrm{nA}\right)$.

\section{RESULTS}

Figure 2 shows the typical characteristics of our devices: the conductance $G$ of the constriction (no SAW applied) and the AE current with respect to the gate voltage. The conductance is corrected for a $0.6 \mathrm{k} \Omega$ series resistance of the contact pads and the 2DEG. Above the pinch-off, $G$ increases smoothly and anomalies that we attribute to conductance plateaus appear at regular intervals. However, the conductance plateaus are considerably below the expected ideal multiples of $G_{0}=2 e^{2} / h$, probably due to additional electron scattering inside the long constriction of our QPCs. ${ }^{17,18}$ Corrections for a larger series resistance would result in an increasing step height between consecutive plateaus. At lower temperatures $(T \ll 2 \mathrm{~K})$ conductance fluctuations appear, indicating the presence of impurities in the vicinity of the constriction.

Applying the SAW results in an AE current, typically starting $\sim 100 \mathrm{mV}$ below conductance pinch-off. The AE current is negative because electrons flow toward the current amplifier (drain) at contact 1, when the measurement configuration of Figs. 1(c) and 1(d) is used. Several plateaus in 


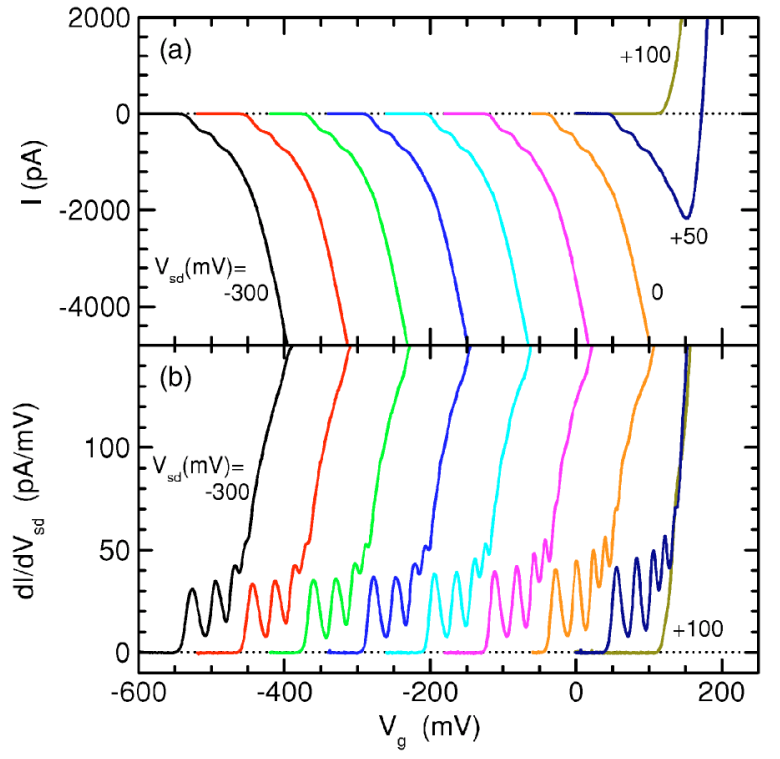

FIG. 3. (Color online) (a) AE current $I$ and (b) conductance $d I / d V_{s d}$ vs gate voltage $V_{g}$. The curves were recorded at fixed bias voltages from -300 to $+100 \mathrm{mV}$ in steps of $50 \mathrm{mV}$ (from left to right). The bias voltage was applied to contact 3 on the same side as the active IDT.

the current at multiples of $e f$ can be seen with the naked eye. Higher-order plateaus can be resolved in the derivative of $I$ versus $V_{g}$. Since all three samples had very similar characteristics, we concentrate in the following on sample $2 \mathrm{C}$.

Figure 3 shows the AE current $I$ and the conductance $d I / d V_{s d}$ with respect to the gate voltage for sample $2 \mathrm{C}$. The data were taken at the optimum rf excitation of this device of $13 \mathrm{dBm}$ and $f=2464.78 \mathrm{MHz}$ at different bias voltages. Plateaus in the $\mathrm{AE}$ current appear near multiples of ef $=395 \mathrm{pA}$. We define the center of the plateau by the minimum slope of either $I\left(V_{s d}\right)$ or $I\left(V_{g}\right)$. A large negative bias voltage at contact 3 only shifts the $I\left(V_{g}\right)$ curves and their derivatives on the gate voltage axis, but does not change their overall shape, as if they were displaced parallel to each other. Such a robustness of $I\left(V_{g}\right)$ against changes in $V_{s d}$ was already demonstrated in Refs. 9 and 10. Deviations occur at sufficiently large positive bias voltages, reversing the polarity of the total current and indicating that a second conduction mechanism develops: tunneling in reverse direction. In the following we operate the QPC in such a transition range between purely acoustoelectric and purely tunneling current.

The AE current plateaus can be observed as well by sweeping the source-drain bias at contact 3 instead of the gate voltage. At sufficiently positive $V_{s d}=V_{+}$, depending on the gate voltage, the current reverses sign and becomes positive within a narrow voltage interval, indicating tunneling through the contact in the direction opposite to the SAW propagation (Fig. 4). All $I\left(V_{s d}\right)$ curves and also their derivatives $d I\left(V_{s d}\right) / d V_{g}$ fall onto single $I\left(V_{s d}\right)$ and $d I / d V_{g}$ curves if properly displaced along the $V_{s d}$ axis. Depending on the chosen displacement, the curves coincide either in the acoustoelectric [Fig. 5(a)] or tunneling regime [Fig. 5(b)]. In both cases the corresponding displacement $\delta V_{s d}$ is proportional to the change in $V_{g}$ but with a different slope (see the inset to Fig. 5).

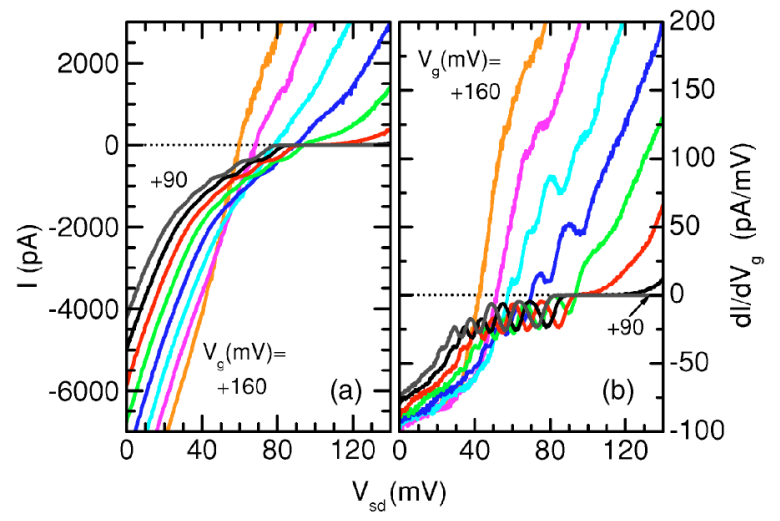

FIG. 4. (Color online) (a) AE current $I$ and (b) transconductance $d I / d V_{g}$ vs bias voltage $V_{s d}$. The $I\left(V_{s d}\right)$ curves were recorded at fixed gate voltages from +90 to $+160 \mathrm{mV}$ in steps of $10 \mathrm{mV}$ as indicated. At $160 \mathrm{mV}$ anomalies due to the quantized AE current can no longer be resolved. The bias voltage was applied to contact 3 on the same side as the active IDT, as in Fig. 3. This explains why the AE current is negative. The positive tunneling counterflow starts at sufficiently positive bias voltages.

Figure 6 shows that biasing contact 1 opposite the active IDT gave similar results, but with reversed polarity. Here the $\mathrm{AE}$ current is positive because the SAW drags electrons out of the drain contact 3 , while the tunneling contribution is negative at negative bias voltages. This time, however, the shifts in bias voltage to match the curves for the acoustoelectric as well as for the tunneling regime are interchanged with respect to the previous case (see the inset to Fig. 7). When contact 1 is biased, adjusting for a match of the AE current requires the same shift, except the sign, as for adjusting the tunneling current when contact 3 is biased and vice versa. This indicates that both shifts are closely related and also that the QPC itself is quite symmetric.

Note the low noise level of our samples. Random telegraph noise, caused by switching of impurity states and typi-

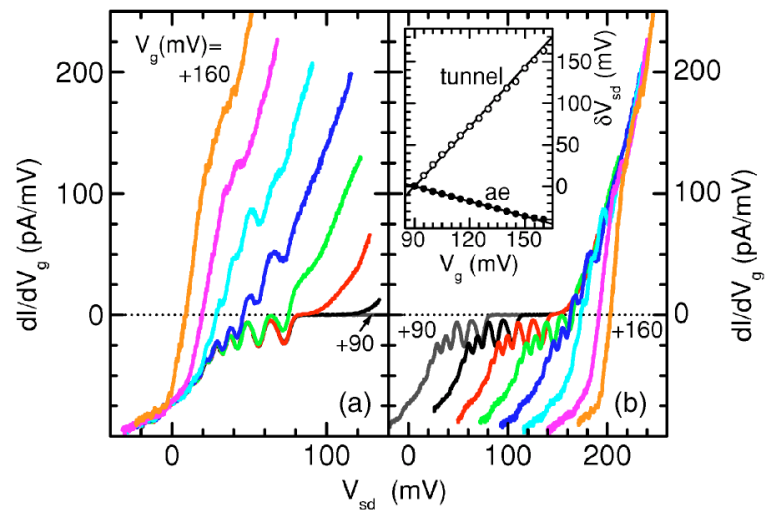

FIG. 5. (Color online) Transconductance $d I / d V_{g}$ vs bias voltage $V_{s d}$. The eight curves from Fig. 4(b) at fixed gate voltages from 90 to $160 \mathrm{mV}$ in steps of $10 \mathrm{mV}$ have been displaced along the bias voltage axis to match (a) at low voltages where the tunneling contribution is small and (b) at large voltages where the AE current is negligible. The required shifts $\delta V_{s d}$ for matching, in the $\mathrm{AE} \mathrm{(ae)} \mathrm{and}$ in the tunneling (tunnel) regime, are shown in the inset. Solid lines guide the eye. The bias voltage was applied to contact 3 near the active IDT. 


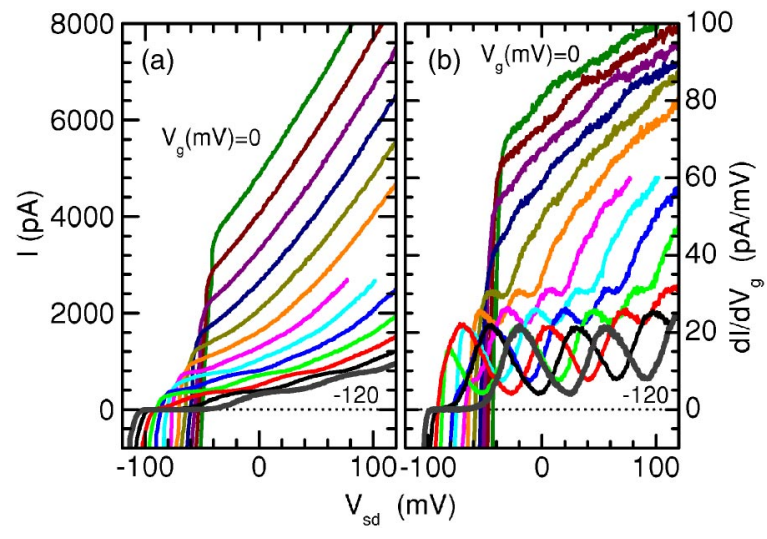

FIG. 6. (Color online) (a) AE current $I$ and (b) transconductance $d I / d V_{g}$ vs bias voltage $V_{s d}$. The $I\left(V_{s d}\right)$ curves were recorded at fixed gate voltages from 0 to $-120 \mathrm{mV}$ in steps of $10 \mathrm{mV}$ as indicated. The bias voltage was applied to contact 1 opposite the active IDT. Therefore, the AE current is positive and the negative tunneling counterflow starts at sufficiently negative bias voltages.

cally of order $e f$, is completely absent. In the acoustoelectric regime we estimate a total current noise of about $1 \mathrm{pA}$ in the $0-1 \mathrm{kHz}$ bandwidth. The noise level increases considerably with growing tunneling counterflow. Since it is much larger than expected for shot noise (spectral density of order $e I$ ), we attribute it to hot-electron effects.

The absolute value of the counterflow is quite small, of order $1 \mathrm{nA}$ at $100 \mathrm{mV}$ bias voltage, corresponding to $10 \mathrm{G} \Omega$. At these conditions heating of the 2DEG due to the

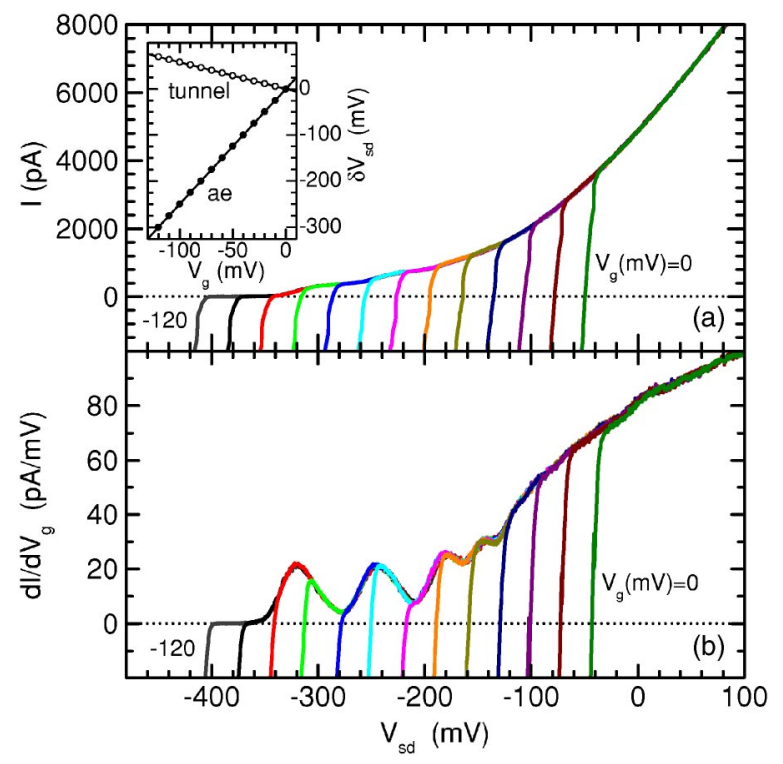

FIG. 7. (Color online) (a) AE current $I$ and (b) transconductance $d I / d V_{g}$ vs bias voltage $V_{s d}$. The 13 curves from Figs. 6(a) and 6(b) at fixed gate voltages from 0 to $-120 \mathrm{mV}$ in steps of $10 \mathrm{mV}$ have been displaced along the bias voltage axis to match at high voltages where the tunneling contribution is small. The corresponding shift $\delta V_{s d}$ is shown in the inset (ae) along with the shift that would be required to match the traces in the tunneling regime (tunnel). Solid lines guide the eye. The bias voltage was applied to contact 1 opposite the active IDT. acoustoelectric or the tunneling current is negligible. To probe heating effects, we used the $2 \mathrm{DEG}$ resistance between contacts 3 and 4 as a built-in temperature sensor. Injecting $100 \mathrm{nA}$ hot electrons at $100 \mathrm{meV}$ from the right into the 2DEG on the left, increased its temperature by less than $0.1 \mathrm{~K}$ at $T \approx 5 \mathrm{~K}$. This estimate is consistent with earlier reports on similar QPC devices. ${ }^{19}$

\section{DISCUSSION}

To qualitatively describe the experiment data, we use the standard model for the QPC potential barrier superposed with the dynamic SAW potential, similar to those in Refs. 12-15. We assume screening of the SAW potential by the 2DEG outside the center of the QPC. For a thorough discussion on screening of the SAW potential see Ref. 20. In addition, one side of the QPC can be biased; that means, its chemical potential can be shifted with respect to the opposite side.

\section{A. Estimating barrier height}

The observed $I\left(V_{s d}\right)$ characteristics are strongly nonlinear due to the current quantization in the $\mathrm{AE}$ regime and the exponential current dependence on bias in the tunneling regime. The onset of tunneling counterflow due to the bias voltage (either with or without the SAW) can be understood as follows. A positive bias at the source contact lowers both the chemical potential $\mu_{s}=\epsilon_{F}-e V_{s d}$ on the source side of the QPC and the barrier height of its lowest subband as indicated in Fig. 1(d). For clarity we set the zero of the energy axis at the bottom of the conduction band on the drain side, kept at virtual ground by the current amplifier. Since the experimental data suggest almost linear relationships in the investigated voltage range, we describe the change in barrier height by $d E / e=-\alpha d V_{s d}$. At a bias voltage $V_{+}$the barrier almost coincides (within a few meV) with the (unshifted) chemical potential $\mu_{d}=\epsilon_{F}$ of the drain side of the QPC; thus, electrons can flow from drain to source. At this condition the difference between the top of the barrier at zero bias $E_{1}$ and the chemical potential on the drain side is $\left(E_{1}-\epsilon_{F}\right) / e=\alpha V_{+}$. A negative bias voltage raises the chemical potential on the source side; but, simultaneously, it also raises the barrier as described above by $d E / e=-\alpha d V_{s d}$. At the critical voltage $V_{-}=-\left(E_{1}-\epsilon_{F}\right) / e+\alpha V_{-}$the chemical potential $\mu_{s}$ reaches the barrier, and electrons start to flow from source to drain. Combining the above two equations yields $\alpha=-V_{-} /\left(V_{+}\right.$ $\left.-V_{-}\right)$. Our sample 2C has $d V_{-}=0.60 \cdot d V_{g}$ and $d V_{+}$ $=-2.50 \cdot d V_{g}$, thus $\alpha=0.194$.

Deviations from the ideal $\alpha=0.50$ can be attributed to the asymmetry introduced by the bias voltage: first, with respect to zero bias the QPC channel on the biased side becomes more narrow at positive bias voltages. This additionally heightens the barrier (self-gating). Second, the bias voltage drops nonuniformly across the QPC. In a classical model the voltage drop depends on the inverse width of the QPC channel. ${ }^{21}$ As a result the position of the potential maximum moves along the QPC channel with the applied bias voltage. 


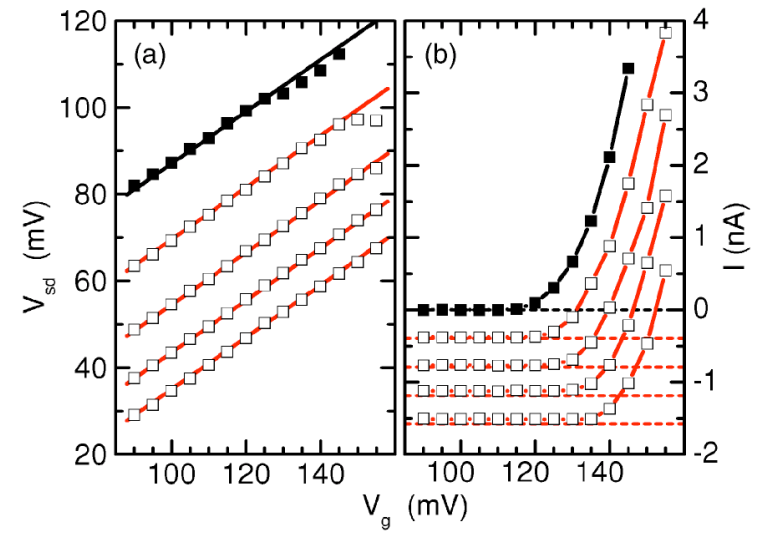

FIG. 8. (Color online) (a) Position of the onset of the AE current (closed symbols) and the first four plateaus at 1-4ef (open symbols, from top to bottom), defined by the transconductance minima in Fig. 4(b). The solid lines through the data points have a slope of 0.60. (b) The actual current at these positions together with the ideal AE current (dashed lines). The bias voltage was applied to contact 3 near the active IDT.

The barrier height changes with gate voltage like $d E / e$ $=-\beta d V_{g}$. For increasing positive biasing, which directly reflects how the barrier changes, the critical voltage $d V_{+}$ $=-2.50 \cdot d V_{g}$. This yields $\beta=+2.50 \cdot \alpha=0.48$ for sample $2 \mathrm{C}$. Using the above estimates for $\alpha$ and $\beta$ we found that at the used rf power, the first acoustoelectric current plateau develops when the Fermi level is about $100 \mathrm{meV}$ below the top of the barrier. At such a condition we estimate, using a hardwall potential model, ${ }^{21}$ a minimum constriction width of less than $10 \mathrm{~nm}$. This ensures that the $\mathrm{AE}$ as well as the tunneling current flow through the same geometrical channel.

\section{B. Separating the two different electrical currents}

Figure 8 summarizes the position of the transconductance minima in Fig. 4 and also the total current at these minima. At small gate voltages the current plateaus approach nearly the expected multiples of $e f$. Deviations from the ideal values are probably due to electron tunneling and thermal activation out of or into the moving quantum dots. ${ }^{12-15}$ Especially thermal activation may play an important role because of the rf-enhanced temperature of the 2DEG. At large gate voltages, counterflow dominates. We can also resolve $\mathrm{AE}$ anomalies while negatively biasing contact 1 . But in this case the tunneling current grows so quickly that it is too difficult to reliably separate the AE current.

The positions of the minima vary linearly both with gate and bias voltage; that is, even at large counterflow they are certainly due to the AE current. The distance between two consecutive $\mathrm{AE}$ current plateaus decreases with growing electron number $n$. However, for two specific plateaus this distance, in terms of gate voltage, does not change when bias voltage is varied and vice versa.

At small counterflow AE current plateaus can still be resolved, but their magnitude seems to be smaller than the theoretical multiples of $e f$. When the counterflow increases, the plateaus can no longer be distinguished in the current while local minima still appear in the transconductance. Thus the AE current is quantized even when there is a large counterflowing current. The maximum counterflow at which an $\mathrm{AE}$ anomaly could be resolved, was about ten times larger then $e f$.

Separating the two contributions to the total current would be straightforward if we could simply switch off the SAW to measure the pure tunneling current. We have found that at fixed gate voltages, such tunneling $I\left(V_{s d}\right)$ characteristics without the SAW have almost the same slope in a semilogarithmic plot as the characteristics with the SAW. They are, however, displaced along the $V_{s d}$-axis as if the SAW lowered the effective barrier. We attribute this to the fact that the operating conditions for the QPC differ markedly in both cases. As mentioned above, the SAW heating changes the 2DEG temperature. Additionally, the shape of the tunneling barrier is altered by the SAW, as indicated schematically in Fig. 1(d) (compare thick and thin solid lines). Thus one should develop an alternative method to determine the tunneling current when the SAW is on.

When tunneling dominates, the $I\left(V_{s d}\right)$ curves as well as their derivatives approach the same asymptotic curves if they are properly displaced on the $V_{s d}$ axis, as shown for the transconductance in Fig. 5(b). We attribute these asymptotes to the counterflowing tunneling current $I_{T}$ and its derivatives $d I_{T} / d V_{g}$ and $d I_{T} / d V_{s d}$. This is the same kind of behavior as found for the $\mathrm{AE}$ current and its derivatives and shown in Fig. 5(a) for the transconductance. Thus, in principle, we know the ideal behavior of the $\mathrm{AE}$ and the tunneling current as well as their derivatives. Therefore it should be possible to separate the two contributions.

For a simple superposition of the two currents, if they would not affect each other, separating the two contributions should recover their ideal values. A preliminary analysis, however, shows that part of the current is missing. If we compare the asymptotic $I_{T}$ with the measured current, the magnitude of the remaining part turns out to be smaller than the magnitude of the expected AE current, which is known from the region with negligible tunneling. The other way round, extrapolating the known $\mathrm{AE}$ regime would result in a larger tunneling current than expected. But we do not know $a$ priori which part is affected most, whether the tunneling current suppresses the AE current, or vice versa, or whether both of them suppress each other.

Assuming $I_{T}$ to be not affected by the AE current, we can calculate the purely acoustoelectric current $I_{A E}=I-I_{T}$, where $I_{T}$ is described by the exponentially dependent asymptote to the experiment data as discussed earlier. To enhance the resolution we use the derivatives instead of the currents, integrating over the difference between the measured $d I / d V_{s d}$ and the asymptotic $d I_{T} / d V_{s d}$ as shown in Fig. 9(a) for one gate voltage. Figure 9(b) displays the AE current obtained in the same way for different gate voltages. One can clearly see that the magnitude of the plateaus decreases for increasing counterflow.

Figure 10 summarizes this kind of analysis for all three samples. For sample $2 \mathrm{~B}$ the $\mathrm{AE}$ current plateaus remain unaffected as long as $I_{T} \leqslant 0.5 \mathrm{nA}$. It is reduced at higher tunneling currents, but saturates at about half of the ideal value when $I_{T} \geqslant 1 \mathrm{nA}$. For sample $2 \mathrm{C}$ the $\mathrm{AE}$ current of the first 


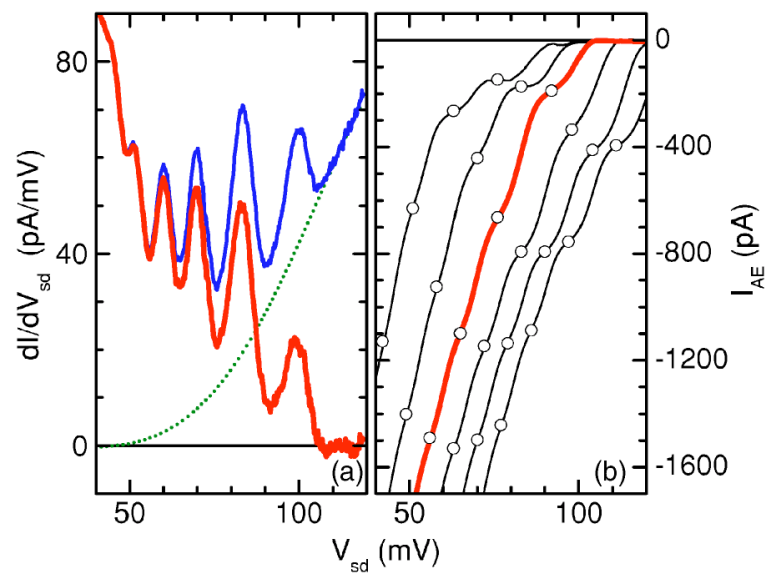

FIG. 9. (Color online) (a) Conductance $d I / d V_{s d}$ vs bias voltage $V_{s d}$ at $V_{g}=135 \mathrm{mV}$ (upper solid line) and the part of the conductance due to tunneling through the QPC (dotted line). The AE conductance contributes then just the difference between the two curves (lower solid line). (b) Thick solid line is the AE current $I_{A E}$ vs bias voltage $V_{s d}$ calculated by integrating the lower solid line in (a). The other curves obtained similarly for gate voltages $V_{g}=145,140 \mathrm{mV}$ and $V_{g}=130,125,120 \mathrm{mV}$ are displaced horizontally in steps of $10 \mathrm{mV}$ to the left and the right, respectively. Open symbols mark the position of the measured conductance minima. The bias voltage was applied to contact 3 near the active IDT.

four plateaus is strongly suppressed even at small tunneling currents. It is reduced linearly up to $I_{T} \approx 0.6 \mathrm{nA}$ and, as it does for sample $2 \mathrm{~B}$, saturates at larger counterflow. This saturation can be unambiguously resolved only for the first and the second plateaus. The relative reduction 1 $-I_{A E}\left(I_{T}\right) / n e f$, however, is the same for each of the $n=1-4$ plateaus. One would expect this if the tunneling current was the dominant one that suppresses the AE current. Sample 2E shows no reduction at all for $n=1$ up to about $I_{T} \approx 4 \mathrm{nA}$. The increase of the AE current for the higher-order plateaus could be an artifact due to the increased uncertainty of the tunnel-

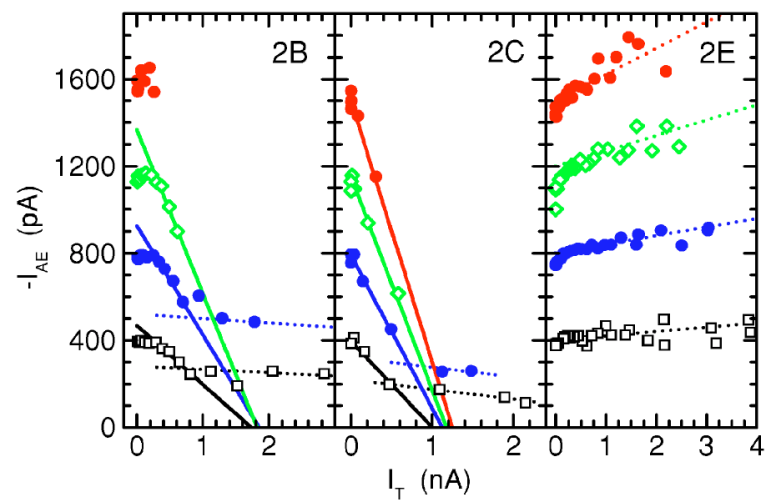

FIG. 10. (Color online) AE current $I_{A E}=I-I_{T}$ at the plateaus vs tunneling counterflow $I_{T}$ for the three investigated samples. For samples $2 \mathrm{~B}$ and $2 \mathrm{C}$, the solid lines indicate a possible convergence of the first four acoustoelectric current plateaus at a common fixed point in tunneling current. The dotted lines represent the asymptotic behavior at large tunneling currents. The bias voltage was applied to contact 3 near the active IDT. ing current. The results of $2 \mathrm{~B}$ might be viewed as a combination of $2 \mathrm{C}$ and $2 \mathrm{E}$ : Starting at small tunneling currents, the AE current first stays constant, as it does for $2 \mathrm{E}$. But at larger tunneling currents, the AE current is suppressed until it saturates, as it does for $2 \mathrm{C}$.

\section{Possible interaction between tunneling and acoustoelectric current}

The simplest explanation for the reduced $\mathrm{AE}$ current (sample 2B and 2C) would be that it is not caused by the counterflow, but by the asymmetric distortion of the static QPC potential barrier due to the bias voltage. Although the moving quantum dots may originally contain an integer number $n$ of electrons, part of them could be lost when the dot travels up the potential barrier, depending on how the barrier is distorted. This would then show up as an enhanced shot noise, which is, nevertheless, difficult to extract in the presence of other noise sources. ${ }^{22}$ However, such an enhanced shot noise would result in a reduced flatness of the AE plateaus. Over a very wide range of bias voltages this is not observed as long as tunneling counterflow is negligible (Fig. 3). Such an effect could be present for sample 2B and $2 \mathrm{C}$, but not for sample $2 \mathrm{E}$, which shows nearly the ideal $\mathrm{AE}$ current even at large counterflow (Fig. 10).

We speculate whether or not the SAW-driven electrons backscatter, with a certain probability, on tunneling electrons. This situation would be similar to the Auger effect observed in quantum-dot devices, ${ }^{23,24}$ indicating a very efficient electron capture and relaxation due to Coulomb scattering. In our case the backscattering probability could be large because both currents have to pass the same $\sim 10 \mathrm{~nm}$ narrow constriction. Electrons trapped in the moving quantum dots have barely any momentum compared to that of the hot electrons moving in opposite direction. Therefore backscattering the tunneling electrons is nearly impossible because that would require a momentum transfer of twice the Fermi momentum. On the other hand, backscattering the SAWtransported electrons would require only a very small momentum transfer, which would not affect the tunneling current. However, the AE current is transported by electrons at the SAW velocity $v_{S A W} \approx 3 \mathrm{~km} / \mathrm{s}$, while the tunneling electrons move much faster at the Fermi velocity $v_{F}$ $\approx 230 \mathrm{~km} / \mathrm{s}$. This implies that it is unlikely for a tunneling electron in the QPC to hit a SAW-transported electron when both currents have similar magnitudes. Thus backscattering should be negligible, in accordance with the results of sample 2E in Fig. 10.

An alternative scenario could be that the tunneling electrons are trapped by the moving quantum dots that already contain one or more electrons. They increase the kinetic energy there, enhance the probability to escape, and, thus, reduce the AE current. This would agree with the reduction of $I_{A E}$ observed for sample $2 \mathrm{~B}$ and $2 \mathrm{C}$. The inelastic mean-free path at $5 \mathrm{~K}$ of $\operatorname{order}^{25} h v_{F} / k_{B} T \approx 2 \mu \mathrm{m}$ is comparable to the length of the QPC channel, therefore, few such processes could happen during the transition time of a tunneling electron. In that case we would expect a strong reduction of $I_{A E}$ at large bias voltages when the inelastic mean-free path is 
further reduced due to the excitation of LO phonons at multiples of $36 \mathrm{meV}$ (see, for example, Ref. 19). However, we could not observe any anomalous behavior of $I_{A E}\left(I_{T}\right)$ at those specific bias voltages. Therefore we consider the reduction of the $\mathrm{AE}$ current due to inelastic scattering of the tunneling electrons at lower bias voltages as negligible.

The different behavior of $I_{A E}\left(I_{T}\right)$ could also reflect the response due to the complex potential landscape of long QPCs, which is not well known. The idealized potential in Fig. 1(d) has a broad maximum due to the QPC constriction, fine modulated by the SAW. But there are reports that instead of a single potential barrier, a double peak could form accidentally, building a quantum dot and enhancing the SAW transport. ${ }^{26}$ Moreover, the effective length of the constriction might be much shorter than its geometrical dimension would suggest. ${ }^{27}$ This certainly needs further investigation.

\section{SUMMARY}

We have studied in detail how two independently injected counterflowing electron currents, one tunneling and the other SAW driven, interact when they pass each other at the QPC. While the AE current can be quantized even at large counterflow, our results indicate that a direct interaction between both currents is negligible. The strongly reduced AE current at large counterflow for two of the samples could instead be due to the bias-induced changes of the potential landscape across the QPC.

\section{ACKNOWLEDGMENT}

We acknowledge support from the European Commission FET Project SAWPHOTON.
${ }^{1}$ Ch. Kittel, Introduction to Solid State Physics, 7th ed. (Wiley, New York, 1996).

${ }^{2}$ T. Hattori, J. Phys. Soc. Jpn. 23, 19 (1967).

${ }^{3}$ H. Linke, T. E. Humphrey, P. E. Lindelof, A. Löfgren, R. Newbury, P. Omling, A. O. Suchkov, R. P. Taylor, and H. Xu, Appl. Phys. A: Mater. Sci. Process. 75, 237 (2002).

${ }^{4}$ I. S. Millard, N. K. Patel, E. H. Linfield, P. D. Rose, M. Y. Simmons, D. A. Ritchie, G. A. C. Jones, and M. Pepper, Semicond. Sci. Technol. 11, 483 (1996).

${ }^{5}$ N. P. R. Hill, J. T. Nicholls, E. H. Linfield, M. Pepper, D. A. Ritchie, A. R. Hamilton, and G. A. C. Jones, J. Phys.: Condens. Matter 8, L557 (1996).

${ }^{6}$ K. Komori, X. L. Wang, M. Ogura, H. Matsuhata, and H. Imanishi, Appl. Phys. Lett. 68, 3787 (1996).

${ }^{7}$ O. M. Auslaender, A. Yacoby, R. de Picciotto, K. W. Baldwin, L. N. Pfeiffer, and K. W. West, Science 295, 825 (2002).

${ }^{8}$ J. M. Shilton, V. I. Talyanskii, M. Pepper, D. A. Ritchie, J. E. F. Frost, C. J. B. Ford, C. G. Smith, and G. A. C. Jones, J. Phys.: Condens. Matter 8, L531 (1996).

${ }^{9}$ V. I. Talyanskii, J. M. Shilton, M. Pepper, C. G. Smith, C. J. B. Ford, E. H. Linfield, D. A. Ritchie, and G. A. C. Jones, Phys. Rev. B 56, 15 (1997).

${ }^{10}$ J. Cunningham, V. I. Talyanskii, J. M. Shilton, M. Pepper, M. Y. Simmons, and D. A. Ritchie, Phys. Rev. B 60, 4850 (1999).

${ }^{11}$ J. Cunningham, V. I. Talyanskii, J. M. Shilton, M. Pepper, A. Kristensen, and P. E. Lindelof, Phys. Rev. B 62, 1564 (2000).

${ }^{12}$ G. Gumbs, G. R. Aizin, and M. Pepper, Phys. Rev. B 60, R13 954 (1999).

${ }^{13}$ P. A. Maksym, Phys. Rev. B 61, 4727 (2000).

${ }^{14}$ A. M. Robinson and C. H. W. Barnes, Phys. Rev. B 63, 165418 (2001).
${ }^{15}$ K. Flensberg, Q. Niu, and M. Pustilnik, Phys. Rev. B 60, R16 291 (1999).

${ }^{16}$ P. Utko, K. Gloos, J. Bindslev Hansen, and P. E. Lindelof, Acta Phys. Pol. A 103, 533 (2003).

${ }^{17}$ B. E. Kane, G. R. Facer, A. S. Dzurak, N. E. Lumpkin, R. G. Clark, L. N. Pfeiffer, and K. W. West, Appl. Phys. Lett. 72, 3506 (1998).

${ }^{18}$ C.-T. Liang, M. Y. Simmons, C. G. Smith, D. A. Ritchie, and M. Pepper, Appl. Phys. Lett. 75, 2975 (1999).

${ }^{19}$ A. S. Dzurak, C. J. B. Ford, M. J. Kelly, M. Pepper, J. E. F. Frost, D. A. Ritchie, G. A. C. Jones, H. Ahmed, and D. G. Hasko, Phys. Rev. B 45, 6309 (1992).

${ }^{20}$ G. R. Aizin, G. Gumbs, and M. Pepper, Phys. Rev. B 58, 10589 (1998).

${ }^{21}$ T. Heinzel, D. A. Wharam, F. M. de Aguiar, J. P. Kotthaus, G. Böhm, W. Klein, G. Tränkle, and G. Weimann, Semicond. Sci. Technol. 9, 1220 (1994).

${ }^{22}$ A. M. Robinson, V. I. Talyanskii, M. Pepper, J. E. Cunningham, E. H. Linfield, and D. A. Ritchie, Phys. Rev. B 65, 045313 (2002).

${ }^{23}$ D. Morris, N. Perret, and S. Fafard, Appl. Phys. Lett. 75, 3593 (1999).

${ }^{24}$ S. Raymond, K. Hinzer, and S. Fafard, Phys. Rev. B 61, R16 331 (2000).

${ }^{25}$ R. Holm, Electric Contacts Handbook, 3rd ed. (Springer, Berlin, 1958).

${ }^{26}$ N. E. Fletcher, J. Ebbecke, T. J. B. M. Janssen, F. J. Ahlers, M. Pepper, H. E. Beere, and D. A. Ritchie, Phys. Rev. B 68, 245310 (2003).

${ }^{27}$ K. Gloos, P. Utko, M. Aagesen, J. Bindslev Hansen, and P. E. Lindelof (unpublished). 